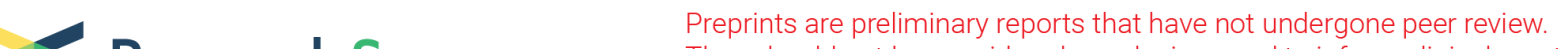 Research Square
They should not be considered conclusive, used to inform clinical practice,
or referenced by the media as validated information.
}

\section{Verrucous carcinoma of the esophagus with complete response after chemoradiotherapy}

\section{Masashi Hashimoto}

"Okayama Daigaku Daigakuin Ishiyakugaku Sogo Kenkyuka Igakubu"

Yasuhiro Shirakawa ( $\square$ yasuwr@md.okayama-u.ac.jp)

"Okayama Daigaku Daigakuin Ishiyakugaku Sogo Kenkyuka Igakubu" https://orcid.org/0000-00034698-5119

\section{Shunsuke Tanabe}

"Okayama Daigaku Daigakuin Ishiyakugaku Sogo Kenkyuka Igakubu"

\section{Takehiro Tanaka}

"Okayama Daigaku Daigakuin Ishiyakugaku Sogo Kenkyuka Igakubu"

\section{Teruki Kobayashi}

"Okayama Daigaku Daigakuin Ishiyakugaku Sogo Kenkyuka Igakubu"

Naoaki Maeda

"Okayama Daigaku Daigakuin Ishiyakugaku Sogo Kenkyuka Igakubu"

\section{Kazuhiro Noma}

"Okayama Daigaku Daigakuin Ishiyakugaku Sogo Kenkyuka Igakubu"

\section{Kazufumi Sakurama}

Shigei Igaku Kenkyujo Fuzoku Byoin

\section{Toshiyoshi Fujiwara}

"Okayama Daigaku Daigakuin Ishiyakugaku Sogo Kenkyuka Igakubu"

\section{Case report}

Keywords: esophagectomy, verrucous carcinoma, esophageal squamous cell carcinoma

Posted Date: June 2nd, 2020

DOI: https://doi.org/10.21203/rs.3.rs-32041/v1

License: (c) (i) This work is licensed under a Creative Commons Attribution 4.0 International License. Read Full License 


\section{Abstract}

\section{Background}

Verrucous carcinoma of the esophagus (VCE) is a rare tumor that is difficult to diagnose. In most cases, biopsies show nonspecific inflammatory and hyperkeratotic changes and do not show malignant findings. Most VCEs are slowly growing, locally advanced tumors with few metastases. Treatments for VCE are the same as for normal esophageal cancer, involving combined chemotherapy, surgical resection, and radiation therapy. However, it has been reported that VCE has a poor response to radiation or chemoradiotherapy (CRT). A case of VCE with complete response (CR) after CRT is presented.

\section{Case presentation}

A 70-year-old man was found to have white, irregular esophageal mucosa four years earlier. He had been followed-up as an outpatient as having candidal esophagitis. However, his tumor grew gradually, and biopsy was performed by endoscopic mucosal resection. He was finally diagnosed with VCE. He had no metastases to distant organs, but some lymph node metastases were suspected. The tumor invaded his left bronchus. First, the esophagostomy and gastrostomy were constructed. The patient then underwent definitive CRT. Four weeks after the end of CRT, two-stage esophagectomy was performed. First, he underwent esophagectomy with thoracic lymph node dissection. A latissimus dorsi flap was patched to the bronchus after primary suture of the hole. Six weeks later, reconstruction of the gastric tube was performed through the antethoracic route. The pathological findings showed complete response to CRT, with no proliferative cancer cells in the specimen. Six months after the first-stage operation, no recurrence has been observed.

\section{Conclusions}

A case of locally advanced VCE that achieved a complete response to CRT was presented. In cases in which local resection would be difficult, CRT might be an appropriate neoadjuvant treatment for VCE.

\section{Background}

Ackerman first reported verrucous carcinoma of the oral cavity in 1948 (1). Although there have been some reports of verrucous carcinoma of various regions, reports of verrucous carcinoma of the esophagus (VCE) have been rare (2). Minielly et al. reported the difficulty of making the diagnosis of VCE (3). The findings of VCE on esophagogastroduodenoscopy (EGD) are a white, warty, plaque-like appearance with superimposed candidal esophagitis $(2,4)$. In most cases, the biopsies show nonspecific inflammatory and hyperkeratotic changes without malignant findings. Most cases of VCE are slowly growing, locally developing tumors with few lymph node or distant metastases (5). However, the delay in making the diagnosis can sometimes be fatal (6). The treatments for VCE are the same as those for common esophageal cancer, involving combined chemotherapy, surgical resection, and radiation therapy. However, it has been reported that VCE has a poor response to radiation or chemoradiotherapy (CRT) (5, 
7). A rare case of VCE with complete response (CR) after CRT proven by the pathological findings of a surgical specimen is presented.

\section{Case Presentation}

A 70-year-old man was referred to our hospital complaining of obstruction that was due to type 3 VCE (MtLtUt, T4bN1M0, cStage IVa, UICC-8) (8). He had no history of smoking and drank alcohol occasionally. He had an EGD examination every year. Although white, irregular esophagealmucosa was found four years earlier, there were no malignant cells in the first biopsy specimen, and candidal esophagitis was diagnosed. He had been followed-up as an outpatient, but stenosis of the esophagus developed gradually. Taking into account his clinical course, endoscopic mucosal resection (EMR) was performed to obtain a large specimen for pathological examination. Histologically, the specimen was a malignant papillary tumor composed of markedly keratinized and well-differentiated squamous cell carcinoma (Fig. 1a). Based on these findings, the lesion was diagnosed as VCE. In our hospital, gastrointestinal endoscopy was performed again, and it showed a tumor with a white, plaque-like, ulcerative appearance, $170 \mathrm{~mm}$ in diameter (Fig. 1b). Computed tomography (CT) and 8Ffluorodeoxyglucose (FDG) positron emission tomography (PET)/CT showed no distant metastases, but two regional lymph node metastases were suspected. Furthermore, CT and bronchoscopy showed that the tumor invaded the left bronchus and made an esophagobronchial fistula (Fig. 2a, b).

The patient's most important problem was that saliva entered his airway continuously, and this caused repeated aspiration pneumonia. First, cervical esophagostomy and percutaneous endoscopic gastrostomy (PEG) were performed as emergent procedures. The aim was to separate the flow of saliva from the airway. He then underwent definitive CRT (2 Gy/30 Fr, total: $60 \mathrm{~Gy}, 2$ cycles of chemotherapy: 5fluorouracil $700 \mathrm{mg} / \mathrm{m}^{2}$ and cisplatin $70 \mathrm{mg} / \mathrm{m}^{2}$ every 4 weeks), and this treatment was very effective. The tumor gradually decreased in size. Finally, although re-examination of the tumor by EGD could not be performed due to the esophagostomy, CT, and FDG-PET-CT showed that the tumor had shrunk dramatically (Fig. 2c), and there were no new metastatic lesions. However, bronchoscopy showed an obvious residual esophagobronchial fistula at the membranous portion of the left bronchus (Fig. 2d). It was decided to perform salvage surgery because the esophagobronchial fistula seemed unlikely to close with conservative treatment, and the remaining tumor could not be completely ruled out. A two-stage operation was performed. In the first-stage surgery, esophagectomy with mediastinal lymph node dissection was performed. After the dissection, the hole due to the fistula in the left bronchus was revealed. After the suturing closure of the hole (Fig. 3a), a latissimus dorsi flap was inserted and patched at the dorsal side of the left bronchus (Fig. 3b, c). Six weeks after the first-stage surgery, the second-stage surgery was performed with reconstruction with a gastric tube through the antethoracic route. The postoperative course was good in both surgeries, and the patient was transferred to another hospital for rehabilitation 21 days after the second-stage operation. The pathological findings showed CR to CRT, with no remaining malignant cells in the surgical specimen (Fig. 3d), and 6 months later, no recurrence was observed. 


\section{Conclusions}

A case of VCE with CR after CRT was reported. Since VCE was first reported in 1968 by Minielly, there have been more than 40 reports of it (3). According to the World Health Organization classification, VCE is defined histologically as a malignant papillary tumor composed of well-differentiated and keratinized squamous epithelium (2). In many reports, VCE was found to be associated with chronic mucosal irritation, achalasia, hiatal hernia, HPV infection, esophagitis, and so on. In the present case, there was no such history $(5,9)$, and the patient did not smoke and drank alcohol only occasionally.

The clinical and pathological diagnosis of VCE is difficult, as in verrucous carcinoma of other organs (4, 10). EGD shows a white, warty, plaque-like appearance with superimposed candidal esophagitis $(2,4)$. Histologically, VCE shows a well-differentiated tumor or often a benign appearance. The superficial layer of VCE is usually covered with hyperkeratosis or acanthosis $(4,10)$. The appearance often resembles that of candidal esophagitis (4). The present case had been followed-up as having candidal esophagitis for four years. Several biopsies did not show malignant cells. It was important to suspect that a case with such an appearance could be VCE. It was also useful to use EMR to obtain a large specimen for pathological examination (4).

Since VCE is a rare tumor, no standard treatment has been established. At present, multimodal therapy comprising surgery, endoscopic resection, chemotherapy, and/or radiotherapy (RT) is administered for VCE. Because of the features of local progression and rare lymph node metastases, endoscopic resection or esophagectomy has been commonly performed $(5,11)$. Generally, RT is not the first-line treatment due to a report that verrucous carcinoma is less radiosensitive than the common type of SCC (7). However, CRT was selected as the first-choice treatment strategy for the present case. Because of the tumor invasion to the left bronchus, it was judged that intensive preoperative therapy was indispensable for the radical esophagectomy without major combined resection of the left trachea. Of course, verrucous carcinoma is less radiosensitive, but it is not radio-resistant (7). Recently, the therapeutic outcomes of CRT for esophageal cancer have improved notably, and a better response might be expected than in the previous reports. Fortunately, since the present case showed a good response to CRT, esophagectomy could be performed to treat the esophagobronchial fistula and the suspected remaining tumor. Furthermore, the pathological findings showed CR to CRT, and no lymph node metastases, similar to other reports.

Due to a specific issue, the esophagobronchial fistula, the present case needed to undergo esophagectomy from the perspective of quality of life. However, it might be controversial whether to undergo surgery or not if without the fistula. However, it was thought that the diagnosis of CR was more difficult in this case of VCE because the diagnosis of CR is difficult even for squamous cell carcinoma, which was known to be radiosensitive (12). Although the response to CRT was good in the present case certainly, most cases reported previously were less radiosensitive. The present case must be followed-up strictly and carefully for a long time if without surgery. Furthermore, stenosis often occurs too after CRT (11). 
A case of locally far advanced VCE in which CRT was very effective and surgery was also useful for an esophagobronchial fistula was presented. CRT might be a neoadjuvant or definitive treatment for advanced VCE. It will be necessary to study more such cases.

\section{Abbreviations}

CR: complete response, CRT: chemoradiotherapy, CT: computed tomography, EGD: esophagogastroduodenoscopy, EMR: endoscopic mucosal resection, FDG: 8F-fluorodeoxyglucose, PEG: percutaneous endoscopic gastrostomy, PET: positron emission tomography, RT: radiotherapy, VCE: verrucous carcinoma of the esophagus

\section{Declarations}

\section{Acknowledgments}

None.

\section{Authors' contributions}

$\mathrm{MH}$ performed the literature review and wrote the manuscript. YS, ST, TT, TK, NM, KS, and KN were involved in the clinical management of the patient. YS participated in the literature review. TF and YS revised the manuscript. All authors were involved in the management of the patient. All authors read and approved the final manuscript.

\section{Funding}

The authors declare that this study was not funded externally.

\section{Availability of data and materials}

Data sharing is not applicable to this article as no datasets were generated or analyzed during the current study.

\section{Ethics approval and consent to participate}

Written informed consent was obtained from the patient for publication of this case report and the accompanying images. Ethics approval is not applicable because this is a case report.

\section{Consent for publication}

Consent for publication was obtained from the patient.

\section{Competing interests}

The authors declare that they have no competing interests. 


\section{References}

1.Ackerman LV. Verrucous carcinoma of the oral cavity. Surgery. 1948;23(4):670-8.

2.Bosman FT, Carneiro F, Hruban RH, Theise ND. WHO classification of tumours of the digestive system: World Health Organization; 2010.

3.Minielly JA, Harrison EG, Jr., Fontana RS, Payne WS. Verrucous squamous cell carcinoma of the esophagus. Cancer. 1967;20(12):2078-87.

4.Sweetser S, Jacobs NL, Wong Kee Song LM. Endoscopic diagnosis and treatment of esophageal verrucous squamous cell cancer. Dis Esophagus. 2014;27(5):452-6.

5.Osborn NK, Keate RF, Trastek VF, Nguyen CC. Verrucous carcinoma of the esophagus: clinicopathophysiologic features and treatment of a rare entity. Dig Dis Sci. 2003;48(3):465-74.

6.Garrard CL, Sheih WJ, Cohn RA, Sawyers JL. Verrucous carcinoma of the esophagus: surgical treatment for an often fatal disease. Am Surg. 1994;60(8):613-6.

7.Ferlito A, Rinaldo A, Mannara GM. Is primary radiotherapy an appropriate option for the treatment of verrucous carcinoma of the head and neck? J Laryngol Otol. 1998;112(2):132-9.

8.Brierley JD, Gospodarowicz MK, Wittekind C. TNM classification of malignant tumours: John Wiley \& Sons; 2016.

9.Tonna J, Palefsky JM, Rabban J, Campos GM, Theodore P, Ladabaum U. Esophageal verrucous carcinoma arising from hyperkeratotic plaques associated with human papilloma virus type 51 . Dis Esophagus. 2010;23(5):E17-20.

10.Devlin S, Falck V, Urbanski SJ, Mitchell P, Romagnuolo J. Verrucous carcinoma of the esophagus eluding multiple sets of endoscopic biopsies and endoscopic ultrasound: a case report and review of the literature. Can J Gastroenterol. 2004;18(7):459-62.

11.Behrens A, Stolte M, Pech O, May A, Ell C. Verrucous Oesophageal Carcinoma: Single Case Report and Case Series Including 15 Patients - Issues for Consideration of Therapeutic Strategies. Viszeralmedizin. 2014;30(5):346-52.

12.Fujishima F, Taniyama Y, Nakamura Y, Okamoto H, Ozawa Y, Ito K, et al. Residual carcinoma cells after chemoradiotherapy for esophageal squamous cell carcinoma patients: striving toward appropriate judgment of biopsy. Dis Esophagus. 2018;31(7).

\section{Figures}




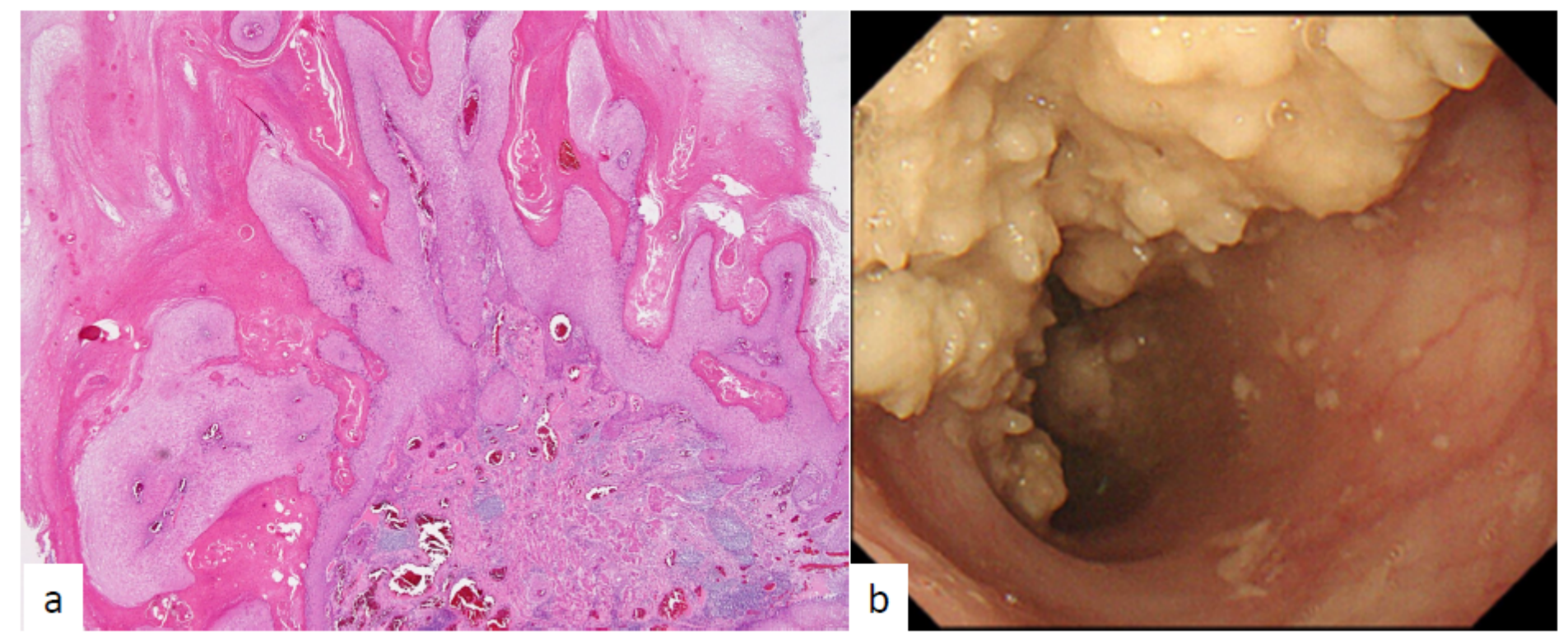

\section{Figure 1}

Images from the histological specimens of previous EMR, and EGD in our hospital. Hematoxylin and eosin staining showed malignant papillary carcinoma composed of keratinized and well-differentiated squamous cell carcinoma (a). EGD showed a white, plaque-like, ulcerative appearance (b). 


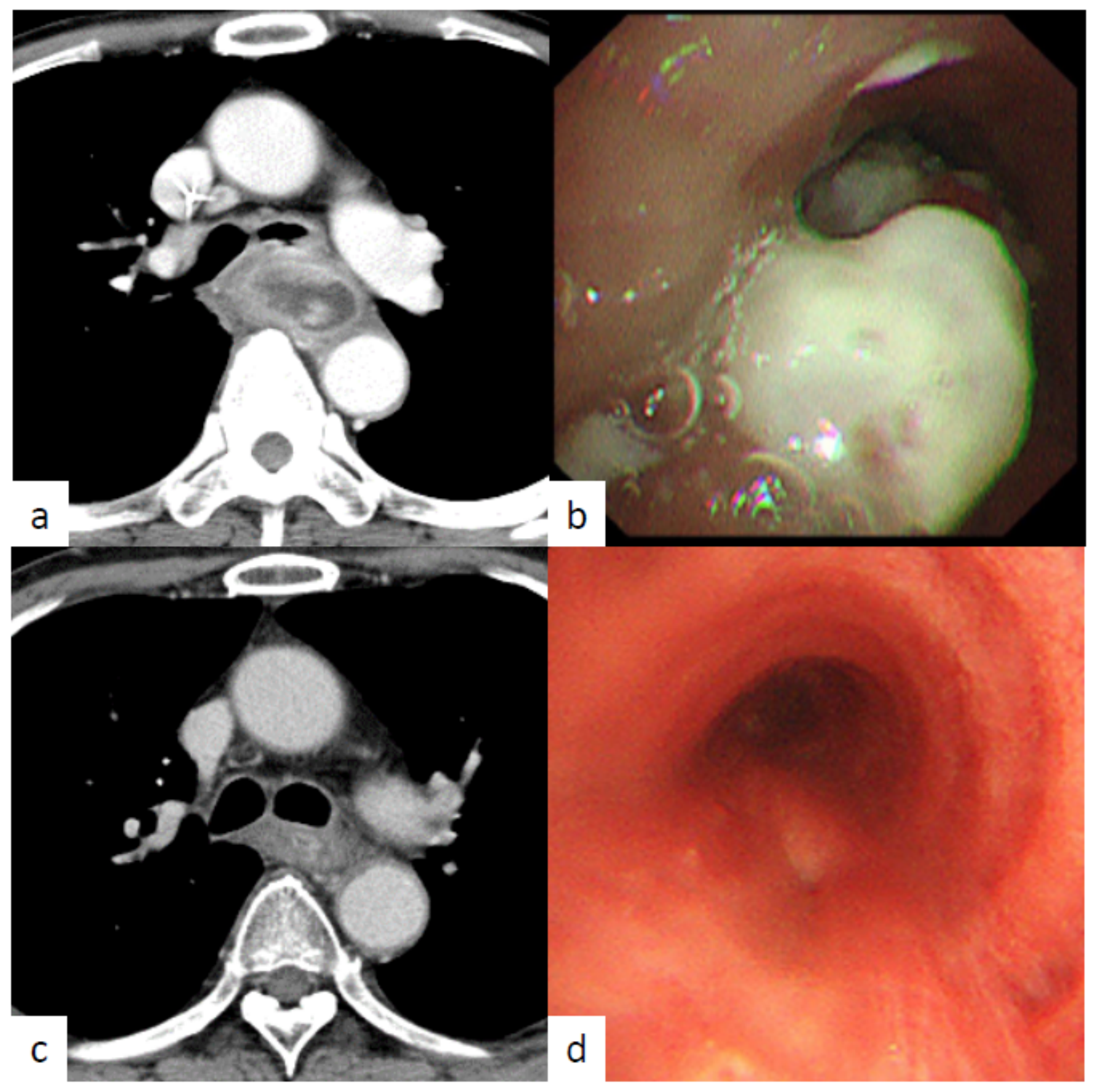

Figure 2

Images from CT and bronchoscopy before and after CRT. Before CRT, CT showed the tumor invading the left bronchus (a) and bronchoscopy showed a white plaque-like erosion at the left bronchus (b). After CRT, CT showed that the tumor has become smaller. There is no evidence of tumor invasion of the bronchus (c), but bronchoscopy showed a residual esophagobronchial fistula (d). 


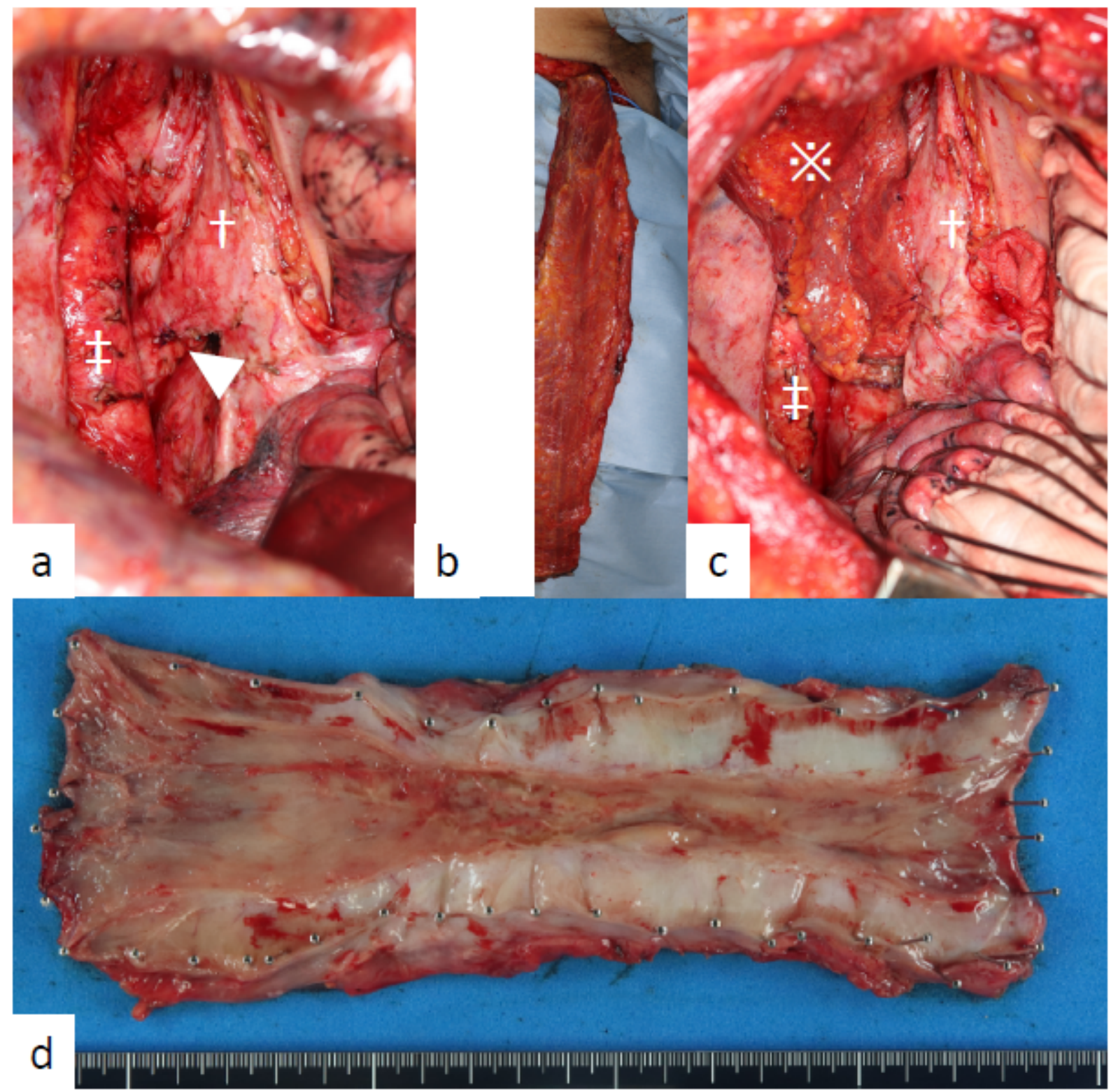

Figure 3

Surgical findings and resected specimen of the esophagus. The hole of the left bronchus after esophagectomy is sutured (arrowhead) (a). Latissimus dorsi flap for patching the hole of the bronchus (b). The latissimus dorsi flap has patched the hole of the left bronchus (c). On gross examination, there is no obvious tumor in the thickening of the esophagus (d). ※: latissimus dorsi flap, tflbronchus, $¥ f$ descending aorta. 